\title{
Table of Contents
}

I. AREAL AND HISTORICAL

1. Vestiges de suffixes de classes nominales dans les langues

du groupe Boua . . . . . . . . . . . . . . . . . . 3

P. Boyeldieu

2. Noun class affix renewal in Southern West Atlantic........ 17

Tucker Childs

3. The expansion of the Gusii tense system . . . . . . . . . . 31 John Kingston

4. Le Rwanda et sa langue . . . . . . . . . . . . . . 57 Léon Mugesera

5. The influence of African languages on pidgins and creoles . . . . 65 John Victor Singler

II. PHONETICS AND PHONOLOGY

6. Geminate consonants in Luganda . . . . . . . . . . . . . 81 Toni Borowsky

7. La construction associative en bété de gbadi ........... 99 Monik Charette

8. A reanalysis of tone in Mende . . . . . . . . . . . . . 127 Patrick Conteh, Elisabeth Cowper, Deborah James, Keren Rice and Michael Szamosi

9. Accent tonal en Kinyarwanda . . . . . . . . . . . . . . 139 Rutinywa Furere et Annie Rialland

10. Pour un traitement synchronique de la faille tonale . . . . . . 151 Gladys Guarisma

11. Are there syllables in Gokana? . . . . . . . . . . . . . 171 Larry M. Hyman

12. La règle d'élision syllabique et les séquences vocaliques en guéré . . . . . . . . . . . . . . . . . 181 Carole Paradis

13. Accent in Kimatuumbi . . . . . . . . . . . . . 195 Douglas Pulleyblank

14. Le systéme tonal du moba comparé à celui du gurma . . . . . . . 217 Annie Rialland 
III. SYNTAX

15. La topicalisation en yoruba . . . . . . . . . . . . . 237 B. R. Badejo

16. Wh-questions and island constraints in Kikuyu:

a reanalysis . . . . . . . . . . . . . . . . . . . 245

Victoria L. Bergvall

17. Wh-in-situ constructions in Egyptian Arabic . . . . . . . . . 261 Michael Kenstowicz and Wafaa Wahba

18. The applied suffix in Kikuyu . . . . . . . . . . . . . 283 Kiyoko Masunaga

19. Bete reciprocals and clitic binding . . . . . . . . . . 292 Dominique Sportiche

IV. SEMANTICS, MORPHOLOGY AND THE LEXICON

20. Dynamique et équilibre du système des pronominaux possessifs en banda-linda . . . . . . . . . . . . . . . . . 319 France Cloarec-Heiss

21. The tense system in Ibibio . . . . . . . . . . . . . . . 329 Okon E. Essien

22. Noun class assignment of English loanwords in Kikuyu . . . . . . 345 Elanah J. Kutik

23. Some nouns and noun phrases in Nzema . . . . . . . . . . . . . 361 William R. Leben

24. Du statut des "concordial elements" dans les langues bantu . . . 369 A. Lipou

25. Spécificité de 1 'Adverbe en Gbaya "bodoe" . . . . . . . . . . . 379 Paulette Roulon 